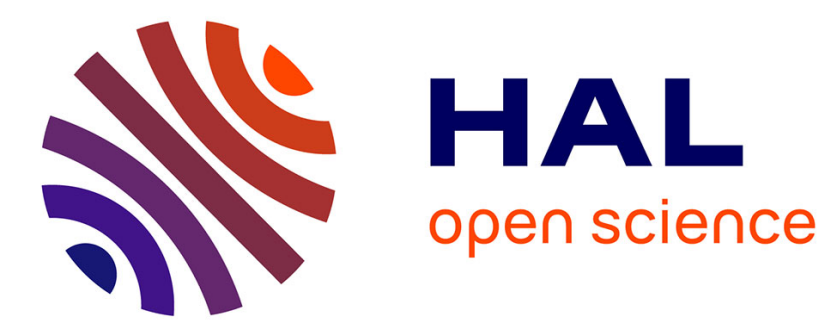

\title{
A Controller Avoiding Dynamic Model Degeneracy of Parallel Robots during Singularity Crossing
}

Damien Six, Sébastien Briot, Abdelhamid Chriette, Philippe Martinet

\section{To cite this version:}

Damien Six, Sébastien Briot, Abdelhamid Chriette, Philippe Martinet. A Controller Avoiding Dynamic Model Degeneracy of Parallel Robots during Singularity Crossing. Journal of Mechanisms and Robotics, 2017, 9 (5), 10.1115/1.4037256 . hal-01524311v2

\section{HAL Id: hal-01524311 \\ https://hal.science/hal-01524311v2}

Submitted on 24 Jun 2019

HAL is a multi-disciplinary open access archive for the deposit and dissemination of scientific research documents, whether they are published or not. The documents may come from teaching and research institutions in France or abroad, or from public or private research centers.
L'archive ouverte pluridisciplinaire HAL, est destinée au dépôt et à la diffusion de documents scientifiques de niveau recherche, publiés ou non, émanant des établissements d'enseignement et de recherche français ou étrangers, des laboratoires publics ou privés. 


\title{
A Controller Avoiding Dynamic Model Degeneracy of Parallel Robots during Singularity Crossing
}

\author{
Damien Six ${ }^{a, b}$ \\ Sébastien Briot ${ }^{\mathrm{a}}$ \\ Abdelhamid Chriette $e^{a, b}$ \\ Philippe Martinet ${ }^{\mathrm{a}, \mathrm{b}}$ \\ a. LS2N, UMR CNRS 6004 \\ b. Ecole Centrale Nantes \\ Nantes 44300 \\ France \\ \{damien.six,sebastien.briot,abdelhamid.chriette,philippe.martinet\}@ls2n.fr
}

\begin{abstract}
Parallel robots present singular configurations that divide the operational workspace into several aspects. It was proven that Type 2 and Leg Passive Joint Twist System singularities can be crossed with a trajectory respecting a given dynamic criterion. However, the practical implementation of a controller able to track such trajectories is up to now limited to restrictive cases of Type 2 singularities crossing. Analyzing the structure of the inverse dynamic model, this paper proposes a global solution allowing the tracking of trajectories respecting the general criterion for any singularity that leads to potential issues of dynamic model degeneracy. The tracking is operated in the robot joint space. Experimental results on a five-bar mechanism showed the controller ability to successfully cross Type 2 singularities.
\end{abstract}

\section{Introduction}

Parallel robots present many advantages over serial robots as higher acceleration capacities, better stiffness and payload-to-weight ratio. However, their use in an industrial context remains limited due, in part, to the division of their workspace in several aspects by singularities [1]. Numerous singularities are prone to appear on a parallel mechanism. For a global overview the reader is referred to [2-4]. In some singularities, the inverse dynamic model of the mechanism does not admit a finite solution without the respect of a dynamic criterion [5]. Those singularities will be referred as Type 2 [6] and Leg Passive Joint Twist System (LPJTS) singularities, which are a kind of leg singularities [4]. The scope of this paper is restricted to the above mentioned singularities. The other singularities such as constraint singularities are not covered.

Several approaches have been explored in the literature to increase the size of the operational workspace of parallel robot, which is usually limited by the presence of singularities. The main approaches are:

- The design of parallel robots without singularities [7-9]. This solution is the most usual one, but it usually leads to the design of robots with a small workspace size or robot architectures with very low practicability.

- The use of redundancy [10-12], which increases the actuation cost.

- Mechanisms with variable actuation modes [13]. This solution is generally carried out when the mechanism is stopped.

- The modification of the Type 2 singularities locus by changing the robot working mode. The main way to proceed is to cross a Type 1 singularity by reaching the workspace boundary and changing the leg configuration [14]. This method however may not grant an access to the complete workspace for all robot architecture.

- The planning of assembly mode changing trajectories encircling cusp points (for Type 2 singularities) [15]. This solution requires the design of complex trajectories, which will not be the most efficient for fast operations, and only few mechanism have cusp points.

- The planning of assembly mode changing trajectories directly through the singularity $[5,16]$ with respect to a dynamic criterion when crossing the singularity.

Crossing the singularity seems a promising solution because a direct path between two points can be generated on any robot structure as long as this path respects the dynamic criterion for crossing. In practical implementation, the precision required on the trajectory tracking is not obtained with a simple PID controller and the effective crossing of the sin- 
gularity is not guaranteed. Then, the use of a dynamic controller as computed-torque control have been explored. Unfortunately, the computation of the inverse dynamic model in such controller require the inversion of matrices that become singular in Type 2 or LPJTS singularities. This issue was partially solved in [17] and [18]. However, both solutions are limited to Type 2 singularities. Also the solution in [17] requires a restrictive trajectories and the solution in [18] is limited to a control of the Cartesian coordinates of the robot tool, which are rarely available on industrial robots.

This paper proposes a global solution allowing the crossing of Type 2 and LPJTS singularities with a computedtorque control, valid for any trajectory that crosses the singularity, as long as the dynamic criterion established in $[5,16]$ is respected. To obtain this solution, the following improvements are performed from existing solutions:

1. The proof that the dynamic criterion expressed in the previous studies is not only a necessary condition to cross the singularity but guarantee a solution to the inverse dynamic model, which is expressed for Type 2 and LPJTS singularities.

2. A projection of the desired wrench when solving the inverse dynamic model in the control law is developed to avoid unbounded input torques from numerical uncertainties and tracking errors.

3. The extension of the second order kinematic equations to singular configurations is obtained to allow a feedback control law in joint space.

The present paper is structured as follows. Section 2 discusses the expression of the inverse dynamic model that remains valid in singularity. Section 3 presents a control law designed to track a trajectory crossing a singularity. The validation of the designed controller is discussed through experimentation in section 4 . Conclusions are given in section 5.

\section{Dynamic model at singularity locus}

\subsection{Dynamic modeling of parallel mechanisms}

In this section, the dynamic equations of a parallel manipulator are briefly recalled. For a more detailed analysis, the reader is referred to $[19,20]$. The studied manipulator is composed of a fixed base, linked by several kinematic chains (the legs) to a mobile platform actuated along $n_{d o f}$ independent coordinates. Actuation is provided by $n_{d o f}$ active joints. $n_{d}$ passive joints are presents in the manipulator legs. The configuration and velocity of the manipulator can be described using:

- $\mathbf{q}_{a}$ and $\dot{\mathbf{q}}_{a}$ two $n_{d o f}$-dimensional vectors of active joint variables and active joint velocities respectively.

- $\mathbf{q}_{d}$ and $\dot{\mathbf{q}}_{d}$ two $n_{d}$-dimensional vectors of passive joint variables and passive joint velocities respectively.

- $\mathbf{x}$ and $\dot{\mathbf{x}}$ two $n_{d o f}$-dimensional vectors of the independent platform coordinates and their time derivative respectively.

The relations between those coordinates are found by writing the closed-loop equations $\mathbf{h}\left(\mathbf{x}, \mathbf{q}_{a}\right)=\mathbf{0}$ and $\mathbf{h}_{a}\left(\mathbf{x}, \mathbf{q}_{a}, \mathbf{q}_{d}\right)=\mathbf{0}$
The time derivative of those closed-loop equations gives the kinematic constraints equations

$$
\begin{aligned}
& \mathbf{A} \dot{\mathbf{x}}+\mathbf{B} \dot{\mathbf{q}}_{a}=\mathbf{0} \\
& \mathbf{J}_{x} \dot{\mathbf{x}}-\mathbf{J}_{a} \dot{\mathbf{q}}_{a}-\mathbf{J}_{d} \dot{\mathbf{q}}_{d}=\mathbf{0}
\end{aligned}
$$

with $\mathbf{A}, \mathbf{B}, \mathbf{J}_{a}, \mathbf{J}_{x}$ and $\mathbf{J}_{d}$ of respective size $\left(n_{d o f} \times n_{d o f}\right)$, $\left(n_{d o f} \times n_{d o f}\right),\left(n_{d} \times n_{d o f}\right),\left(n_{d} \times n_{d o f}\right)$ and $\left(n_{d} \times n_{d}\right)$. Let us also consider the Lagrangian $L$ of the system and $\tau_{a}, \tau_{d}$ and $\mathbf{w}_{x}$ respectively $n_{d o f}, n_{d}$ and $n_{d o f}$-dimensional vectors related to the Lagrangian by

$$
\begin{aligned}
\tau_{a} & =\frac{\mathrm{d}}{\mathrm{dt}}\left(\frac{\partial L}{\partial \dot{\mathbf{q}}_{a}}\right)^{T}-\left(\frac{\partial L}{\partial \mathbf{q}_{a}}\right)^{T} \\
\tau_{d} & =\frac{\mathrm{d}}{\mathrm{dt}}\left(\frac{\partial L}{\partial \dot{\mathbf{q}}_{d}}\right)^{T}-\left(\frac{\partial L}{\partial \mathbf{q}_{d}}\right)^{T} \\
\mathbf{w}_{x} & =\frac{\mathrm{d}}{\mathrm{dt}}\left(\frac{\partial L}{\partial \dot{\mathbf{x}}}\right)^{T}-\left(\frac{\partial L}{\partial \mathbf{x}}\right)^{T}
\end{aligned}
$$

Using method of Lagrange multipliers, the inverse dynamic model of the mechanism can be written as [20]

$$
\begin{aligned}
& \tau=\tau_{a}-\mathbf{B}^{T} \lambda_{1}-\mathbf{J}_{a}^{T} \lambda_{2} \\
& \mathbf{A}^{T} \lambda_{1}-\mathbf{J}_{x}^{T} \lambda_{2}=\mathbf{w}_{x} \\
& \mathbf{J}_{d}^{T} \lambda_{2}=\tau_{d}
\end{aligned}
$$

\section{where}

- $\tau$ is the $n_{d o f}$-dimensional vector of the input efforts,

- $\lambda_{1}$ and $\lambda_{2}$ are a $n_{d o f}$-dimensional and $n_{d}$-dimensional vectors of Lagrange multipliers respectively,

From the expression of the Lagrangian of the mechanical system, equations (3)-(5) can be obtained under the form [20]

$$
\begin{aligned}
\tau_{a} & =\mathbf{M}_{a}\left[\begin{array}{c}
\ddot{\mathbf{q}}_{a} \\
\ddot{\mathbf{q}}_{d}
\end{array}\right]+\mathbf{c}_{a} \\
\tau_{d} & =\mathbf{M}_{d}\left[\begin{array}{c}
\ddot{\mathbf{q}}_{a} \\
\ddot{\mathbf{q}}_{d}
\end{array}\right]+\mathbf{c}_{d} \\
\mathbf{w}_{x} & =\mathbf{M}_{x} \ddot{\mathbf{x}}+\mathbf{c}_{x}
\end{aligned}
$$

where

- $\mathbf{M}_{a}$ is a $\left(n_{d o f} \times\left(n_{d o f}+n_{p}\right)\right)$ matrix and $\mathbf{M}_{d}$ a $\left(n_{p} \times\right.$ $\left.\left(n_{d o f}+n_{p}\right)\right)$ matrix depending on the joint coordinates $\mathbf{q}_{a}$ and $\mathbf{q}_{d}$,

- $\mathbf{c}_{a}$ is a $n_{d o f}$-dimensional vector and $\mathbf{c}_{d}$ a $n_{d}$-dimensional vector depending on the joint coordinates $\mathbf{q}_{a}$ and $\mathbf{q}_{d}$ and velocities $\dot{\mathbf{q}}_{a}$ and $\dot{\mathbf{q}}_{d}$,

- $\mathbf{M}_{x}$ is a $\left(n_{d o f} \times n_{d o f}\right)$ definite positive matrix depending on the platform coordinates $\mathbf{x}$,

- $\mathbf{c}_{r}$ a $n_{d o f}$-dimensional vector depending on the platform coordinates $\mathbf{x}$ and their time derivative $\dot{\mathbf{x}}$ 


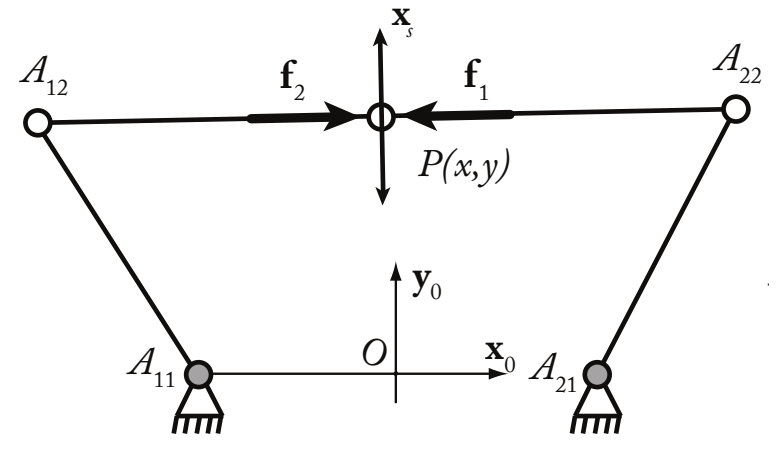

Figure 1: Example of a five-bar mechanism in a Type 2 singularity configuration. The vector $\dot{\mathbf{x}}_{s}$ represents the direction of the uncontrolled platform motion. $\mathbf{f}_{1}$ and $\mathbf{f}_{2}$ are the forces that can be generated through actuation on the platform. In this configuration, the resultant force that can be applied by the system on the platform is collinear to $\mathbf{f}_{1}$ and $\mathbf{f}_{2}$. Two actuators are used to generate an effort along one direction, an overconstraint is consequently generated in the mechanism.

\subsection{Solution to the inverse dynamic model and degener- acy conditions}

In this section, the main results concerning the general solution to the inverse dynamic model equations (6)-(8) and the degeneracy conditions of this model are recalled. For a detailed study of the dynamic model and its degeneracy conditions, the reader is referred to [5]. The general solution to the inverse dynamic model of a parallel robot can be computed by expressing $\lambda_{2}$ from (8) and $\lambda_{1}$ from (7) and by introducing them into (6)

$$
\tau=\tau_{a}-\mathbf{J}_{a}^{T} \mathbf{J}_{d}^{-T} \tau_{d}-\mathbf{B}^{T} \mathbf{A}^{-T} \mathbf{w}_{d}
$$

with $\mathbf{w}_{d}=\mathbf{w}_{x}+\mathbf{J}_{x}^{T} \mathbf{J}_{d}^{-T} \tau_{d}$. The expression of the dynamic model under this form requires a full rank of the matrices $\mathbf{A}$ and $\mathbf{J}_{d}$ [5]. However, this requirement is not always met. The rank degeneracy of those matrices is associated to kinematic singularities of parallel mechanisms identified in the literature:

- The degeneracy of the matrix A corresponds to a kinematic singularity named Type 2 singularity [6]. In such singularity, one (or more) of the degrees of freedom of the platform becomes uncontrollable (see Fig. 1).

- The degeneracy of the matrix $\mathbf{J}_{d}$ corresponds to a kinematic singularity named LPJTS singularity $[4,20]$. In such singularity, one (or more) of the leg gains an uncontrollable motion on a passive joint, even if the platform is fully controlled.

For clarity purposes, it is assumed that only one direction is uncontrollable at singularity locus, corresponding to rank degeneracy of the matrix $\mathbf{A}$ or $\mathbf{J}_{d}$ of order one ${ }^{1}$. It was previously proved that the inverse dynamic model remains consistent under the respect of a dynamic criterion when cross-

\footnotetext{
${ }^{1}$ Even if the case rarely appears on existing parallel robots, the results can be extended to to a higher order degeneracy.
} Paper JMR-17-1046 - Corresponding Author: SIX
Table 1: Dynamic criterion to avoid degeneracy of the inverse dynamic model in a singularity

\begin{tabular}{c|c|c} 
Singularity & Type 2 [21] & LPJTS [5] \\
\hline \hline Criterion & $\dot{\mathbf{x}}_{s}^{T} \mathbf{w}_{d}=0$ & $\dot{\mathbf{q}}_{d s}^{T} \tau_{d}=0$ \\
& $\dot{\mathbf{x}}_{s} \in \operatorname{ker}(\mathbf{A})$ & $\dot{\mathbf{q}}_{d s} \in \operatorname{ker}\left(\mathbf{J}_{d}\right)$
\end{tabular}

ing a singularity. The criteria established are summarized in Table 1. Proofs and considerations on those criteria can be found in [21] and extended to LPJTS singularities in [5].

\subsection{Solution to the inverse dynamic model at singularity locus}

Equation (12) gives a solution to the dynamic model away from singularities. In this section, a solution to the inverse dynamic model in a singularity is given under the respect of the criteria described in Table 1.

\subsubsection{Type 2 singularity}

Let us assume a parallel robot on a Type 2 singularity with no other singularity on the same locus ${ }^{2}$. Then, the inverse dynamic equations (6)-(8) can be written under the form

$$
\begin{aligned}
& \tau=\tau_{a}-\mathbf{B}^{T} \lambda_{1}-\mathbf{J}_{a}^{T} \mathbf{J}_{d}^{-T} \tau_{d} \\
& \mathbf{A}^{T} \lambda_{1}=\mathbf{w}_{d}
\end{aligned}
$$

with $\mathbf{w}_{d}=\mathbf{w}_{x}+\mathbf{J}_{x}^{T} \mathbf{J}_{d}^{-T} \tau_{d}$. The rank degeneracy of the matrix $\mathbf{A}$ implies that the equation (14) will give at least one exact solution $\lambda_{1}$ if and only if $\mathbf{w}_{d}$ is included in the range of the matrix $\mathbf{A}^{T}$. The range of $\mathbf{A}^{T}$ represents all the total wrenches $\mathbf{w}_{d}$ that can be applied by the legs through actuation and external forces on the platform. From linear algebra, it is known that the range of the matrix $\mathbf{A}^{T}$ is the orthogonal complement of the kernel of the matrix $\mathbf{A}$

$$
\operatorname{ker}(\mathbf{A})^{\perp}=\operatorname{Im}\left(\mathbf{A}^{T}\right)
$$

If the dynamic criterion expressed in table 1 is respected, then $\mathbf{w}_{d}$ is in the range of the matrix $\mathbf{A}$ and the inverse dynamic equations admits at least an exact solution. In order to find it, the inverse dynamic model is projected on the platform efforts by pre-multiplying equation (13) by $\mathbf{A}^{T} \mathbf{B}^{-T}$

$$
\mathbf{A}^{T} \mathbf{B}^{-T} \tau=\mathbf{A}^{T} \mathbf{B}^{-T} \tau_{a}-\mathbf{A}^{T} \lambda_{1}-\mathbf{A}^{T} \mathbf{B}^{-T} \mathbf{J}_{a}^{T} \mathbf{J}_{d}^{-T} \tau_{d}
$$

Then, $\mathbf{A}^{T} \lambda_{1}$ can be replaced in (16) from (14), giving

$$
\mathbf{J}_{T 2}^{T} \tau=\mathbf{w}_{T 2}
$$

\footnotetext{
${ }^{2}$ This hypothesis is taken given that the case of the coincidence of two singularities is extremely rare and generally avoided in the design of a parallel robot.
} 
with $\mathbf{J}_{T 2}=\mathbf{B}^{-1} \mathbf{A}$ and

$\mathbf{w}_{T 2}=\mathbf{J}_{T 2}^{T} \tau_{a}-\mathbf{w}_{x}-\left(\mathbf{J}_{x}^{T}+\mathbf{J}_{T 2}^{T} \mathbf{J}_{a}^{T}\right) \mathbf{J}_{d}^{-T} \tau_{d}$.

Knowing that a solution to the inverse dynamic model (13)-

(14) is garanteed if the dynamic criterion is respected, one solution can be expressed using the Moore-Penrose pseudoinverse

$$
\tau=\mathbf{J}_{T 2}^{T}{ }^{+} \mathbf{w}_{T 2}
$$

Since the matrix $\mathbf{J}_{T 2}^{T}$ is not full rank, its kernel is not empty, and so there is an infinite number of solutions to the inverse dynamic model including (18). An illustration is given Fig. 1, on a five-bar mechanism. The wrenches generated by the system on the platform at singularity are two collinear forces. Their opposite action generates an overconstraint in the system. Several combination of those forces (and so several input torques) can generate a desired acceleration of the platform. Equation (18) gives the solution to the inverse dynamic model minimizing the input torques, which is usually the desired solution to limit the power consumption of the mechanical system. However, for specific applications, it is possible to express a general solution to the inverse dynamic model under the form

$$
\boldsymbol{\tau}=\mathbf{J}_{T 2}^{T}{ }^{+} \mathbf{w}_{T 2}+\left(\mathbf{I}_{n_{d o f}}-\mathbf{J}_{T 2}^{T}{ }^{+} \mathbf{J}_{T 2}^{T}\right) \nu
$$

with $\nu$ a $n_{d o f}$-dimensional arbitrary vector called the overconstraint. Among the possible solutions to the inverse dynamic model, the solution minimizing the input torques, given by equation (18), will be retained. Note also that, out of a singularity, the following equality is obtained $\mathbf{J}_{T 2}^{T}{ }^{+}=$ $\mathbf{J}_{T 2}^{-T}$. Then, the solution to the inverse dynamic model under the form (18) is also valid out of the singularity (equivalent to equation (12)).

\subsubsection{LPJTS singularity}

An equivalent inverse dynamic model can be expressed for a LPJTS singularity. In this section, a solution to the inverse dynamic model on a LPJTS singularity is expressed under the respect of the criterion given in Table 1. The inverse dynamic equations (6)-(8) can be written under the form

$$
\begin{aligned}
& \tau=\tau_{a}-\mathbf{B}^{T} \mathbf{A}^{-T} \mathbf{w}_{x}-\mathbf{J}_{d a}^{T} \boldsymbol{\lambda}_{2} \\
& \mathbf{J}_{d}^{T} \boldsymbol{\lambda}_{2}=\tau_{d}
\end{aligned}
$$

with $\mathbf{J}_{d a}=\mathbf{J}_{x} \mathbf{A}^{-1} \mathbf{B}+\mathbf{J}_{a}$. Note that $\mathbf{J}_{d a}$ is a Jacobian matrix linking the velocity of the active joints to the velocity of the passive joints

$$
\mathbf{J}_{d a} \dot{\mathbf{q}}_{a}+\mathbf{J}_{d} \dot{\mathbf{q}}_{d}=\mathbf{0}
$$

The degeneracy of the matrix $\mathbf{J}_{d a}$ corresponds to a rare singularity where the mechanism can generate a non-null active joint velocity $\dot{\mathbf{q}}_{a}$ with no motion of the passive joints. Let us consider that the LPJTS singularity does not coincide with such singularity or a Type 2 singularity ${ }^{3}$. The rank degeneracy of the matrix $\mathbf{J}_{d}^{T}$ implies that the equation (21) admits at least one exact solution $\lambda_{2}$ if and only if $\tau_{d}$ is included in the range of $\mathbf{J}_{d}^{T}$ which is an equivalent condition to the criterion previously defined to cross a LPJTS singularity in Table 1. Projecting the inverse dynamic model (20)-(21) on the efforts exerted on the passive joints gives

$$
\mathbf{J}_{p}^{T} \tau=\mathbf{w}_{p}
$$

with $\mathbf{J}_{p}=\mathbf{J}_{d a}^{-1} \mathbf{J}_{d}$ and

$\mathbf{w}_{p}=-\tau_{d}+\mathbf{J}_{d}^{T} \mathbf{J}_{d a}^{-T}\left(\tau_{a}-\mathbf{B}^{T} \mathbf{A}^{-T} \mathbf{w}_{x}\right)$

With the respect of the criterion (21), the exact solution to the inverse dynamic model minimizing the input torques is

$$
\tau=\mathbf{J}_{p}^{T+} \mathbf{w}_{p}
$$

As in the case of a Type 2 singularity, the general solution to the inverse dynamic model (20)-(21) can be expressed under the form

$$
\tau=\mathbf{J}_{p}^{T+} \mathbf{w}_{p}+\left(\mathbf{I}_{n_{d o f}}-\mathbf{J}_{p}^{T+} \mathbf{J}_{p}^{T}\right) \nu
$$

with $\nu$ an arbitrary vector of overconstraint.

\section{Control law}

In this section, the design of a computed torque control law in order to track trajectories crossing a singularity is discussed. As industrial robot sensors usually measure the active joint coordinates, the control designed is based on the joint coordinates of the mechanical system.

\subsection{Extended second-order loop-closure equations in the neighborhood of a singularity}

The inverse dynamic model of a parallel mechanism contains terms function of the platform, passive and active joints accelerations. To design a computed torque control in joint space, it is necessary to express those accelerations as function of the controlled coordinates acceleration, i.e. the active joint accelerations. The time derivative of loopclosure equations (1) and (2) gives

$$
\begin{aligned}
& \mathbf{A} \ddot{\mathbf{x}}+\mathbf{B} \ddot{\mathbf{q}}_{a}+\mathbf{b}=\mathbf{0} \\
& \mathbf{J}_{x} \ddot{\mathbf{x}}-\mathbf{J}_{a} \ddot{\mathbf{q}}_{a}-\mathbf{J}_{d} \ddot{\mathbf{q}}_{d}+\mathbf{d}=\mathbf{0}
\end{aligned}
$$

with $\mathbf{b}=\dot{\mathbf{A}} \mathbf{t}_{r}+\dot{\mathbf{B}} \dot{\mathbf{q}}_{a}$ and $\mathbf{d}=\dot{\mathbf{J}}_{x} \dot{\mathbf{x}}-\dot{\mathbf{J}}_{t a} \dot{\mathbf{q}}_{a}-\dot{\mathbf{J}}_{t d} \dot{\mathbf{q}}_{d}$. Out of a Type 2 singularity, those two equations can be combined

\footnotetext{
${ }^{3}$ This hypothesis is taken given that the case of the coincidence of two singularities is extremely rare and generally avoided in the design of a parallel robot.
} 
giving

$$
\mathbf{J}_{d a} \ddot{\mathbf{q}}_{a}+\mathbf{J}_{d} \ddot{\mathbf{q}}_{d}+\mathbf{n}=\mathbf{0}
$$

with $\mathbf{J}_{d a}=\mathbf{J}_{x} \mathbf{A}^{-1} \mathbf{B}+\mathbf{J}_{a}$ and $\mathbf{n}=\mathbf{J}_{x} \mathbf{A}^{-1} \mathbf{b}-\mathbf{d}$. Then, out of singularities, the passive joints acceleration and platform acceleration can be expressed as function of the active joint acceleration with

$$
\begin{aligned}
\ddot{\mathbf{x}} & =-\mathbf{A}^{-1} \mathbf{B} \ddot{\mathbf{q}}_{a}-\mathbf{A}^{-1} \mathbf{b} \\
\ddot{\mathbf{q}}_{d} & =-\mathbf{J}_{d}^{-1} \mathbf{J}_{d a} \ddot{\mathbf{q}}_{a}-\mathbf{J}_{d}^{-1} \mathbf{n}
\end{aligned}
$$

However, those expressions are not valid in singularities, as the matrices $\mathbf{A}$ and $\mathbf{J}_{d}$ are singular. To compute those expressions in singularity, it is necessary to extend the second-order loop-closure equations.

\subsubsection{Type 2 singularity}

When crossing a Type 2 singularity the inversion of the matrix A required to solve equation (26) is not possible anymore. In a Type 2 singularity the appearance of one uncontrolled degree of freedom on the platform is related to a kinematic constraint on the joint coordinates. This constraint is respected if and only if $-\mathbf{B} \ddot{\mathbf{q}}_{a}-\mathbf{b}$ is included in the range of A. Let us consider this constraint respected, then equation (26) will give an infinite number of solutions

$$
\ddot{\mathbf{x}}=-\mathbf{A}^{+}\left(\mathbf{B} \ddot{\mathbf{q}}_{a}+\mathbf{b}\right)+\left(\mathbf{I}_{n_{a}}-\mathbf{A}^{+} \mathbf{A}\right) \nu
$$

with $\nu$ a $n_{a}$-dimensional arbitrary vector. This is due to the fact that the platform coordinates acceleration vector $\ddot{\mathbf{x}}$ do not only depends on joint accelerations but also on free dynamics associated to the uncontrollable platform motion. The dynamic criterion expressed in Table 1

$$
\dot{\mathbf{x}}_{s}^{T} \mathbf{w}_{d}=0
$$

is not only a necessary criterion to be respected by a trajectory that crosses the singularity but also an expression of the free dynamics of the platform. This free dynamics can be developed as function of $\ddot{\mathbf{x}}$. Let us consider the Lagrangian of the robot as function of the active joint and platform coordinates and velocities $L_{a x}=f\left(\dot{\mathbf{q}}_{a}, \mathbf{q}_{a}, \dot{\mathbf{x}}, \mathbf{x}\right)$. Then $\mathbf{w}_{d}$ is given by

$$
\begin{aligned}
\mathbf{w}_{d} & =\frac{\mathrm{d}}{\mathrm{dt}}\left(\frac{\partial L_{a x}}{\partial \dot{\mathbf{x}}}\right)^{T}-\left(\frac{\partial L_{a x}}{\partial \mathbf{x}}\right)^{T} \\
& =\mathbf{M}_{a x} \ddot{\mathbf{x}}+\mathbf{c}_{a x}
\end{aligned}
$$

with $\mathbf{M}_{a x}$ a definite positive matrix $\left(n_{a} \times n_{a}\right)$. Equation (33) introduced in equation (32) gives

$$
\dot{\mathbf{x}}_{s}^{T} \mathbf{M}_{a x} \ddot{\mathbf{x}}+\dot{\mathbf{x}}_{s}^{T} \mathbf{c}_{a x}=0
$$

The second-order loop-closure equation (26) can be completed with equation (34) giving

$$
\mathbf{A}_{e} \ddot{\mathbf{x}}=-\mathbf{B}_{e} \ddot{\mathbf{q}}_{a}-\mathbf{b}_{e}
$$

with

$$
\mathbf{A}_{e}=\left[\begin{array}{c}
\mathbf{A} \\
\dot{\mathbf{x}}_{s}^{T} \mathbf{M}_{a x}
\end{array}\right] \quad \mathbf{B}_{e}=\left[\begin{array}{c}
\mathbf{B} \\
\mathbf{0}_{1 \times n_{d o f}}
\end{array}\right] \quad \mathbf{b}_{e}=\left[\begin{array}{c}
\mathbf{b} \\
\dot{\mathbf{x}}_{s}^{T} \mathbf{c}_{a x}
\end{array}\right]
$$

$\mathbf{A}_{e}$ is a $\left(n_{d o f}+1 \times n_{d o f}\right)$ matrix. Even at singularity, matrix $\mathbf{A}_{e}$ rank remains equal to $n_{\text {dof }}$ (see proof below). If a solution to this system exists, it is unique and given by the expression

$$
\ddot{\mathbf{x}}=-\mathbf{A}_{e}^{+}\left(\mathbf{B}_{e} \ddot{\mathbf{q}}_{a}+\mathbf{b}_{e}\right)
$$

The existence of the solution can be guaranteed for a trajectory designed in Cartesian space and then mapped to joint space. However, when using this expression in the controller design, the existence of an exact solution is not guaranteed. The computation of the solution through the MoorePenrose pseudo-inverse will ensure the computation of a solution minimizing the quadratic error in $\ddot{\mathbf{x}}$, thus leading to a good estimation of the inverse dynamic model.

Proof. The solutions to equation (26) expressed in equation (31) span an affine subspace $S$ of the platform accelerations of direction $\operatorname{ker} \mathbf{A}$ (considered dimension 1). We will prove that the affine subspace spanned by the solutions in $\ddot{\mathbf{x}}$ to the dynamic criterion equation (34) intersect in one unique point with $\mathcal{S}$. The solutions to equation (34) is an affine subspace of dimension $n_{d o f}-1$. The direction of this affine subspace is given by

$$
\dot{\mathbf{x}}_{s}^{T} \mathbf{M}_{a x} \ddot{\mathbf{x}}=0
$$

As $\mathbf{M}_{a x}$ is a positive definite matrices, $\dot{\mathbf{x}}_{s}^{T} \mathbf{M}_{a x} \dot{\mathbf{x}}_{s}>0$. Thus, any $\dot{\mathbf{x}}_{s}$ in ker $\mathbf{A}$ is not solution to the equation (37), meaning that $\operatorname{ker} \mathbf{A}$ is not included in the direction given by (37). Then, the affine subspaces spanned by the solutions in $\dot{\mathbf{x}}$ to the equations (26) and (34) are two supplementary subspaces. Their intersection is a point in the platform accelerations space. This point is the unique possible platform acceleration that can be performed by the robot in the singularity, completing the proof.

The solution to the extended loop-closure equations (35) gives an exact computation of the platform acceleration if and only if the dynamic criterion is respected by the platform acceleration vector. However, in the design of our trajectories, the criterion is only respected in the neighborhood of a singularity. Away from it, it will be necessary to use the classical loop-closure equations (26). For the purpose of designing a controller in experimental conditions, the transition between those two equations have to be continuous in 
the neighborhood of the singularity. A Type 2 singularity is characterized by the degeneracy of the matrix $\mathbf{A}$. The matrix condition number $\kappa(\mathbf{A})=\frac{e_{\max }(\mathbf{A})}{e_{\min }(\mathbf{A})}$ is chosen as an indicator of the proximity to the singularity, with $e_{\max }()$ and $e_{\min }()$ being respectively the maximal and minimal singular values of a matrix, leading to

$$
\begin{aligned}
\ddot{\mathbf{x}} & =-\left(\sigma \mathbf{A}^{-1} \mathbf{B}+(1-\sigma) \mathbf{A}_{e}^{+} \mathbf{B}_{e}\right) \ddot{\mathbf{q}}_{a} \\
& -\sigma \mathbf{A}^{-1} \mathbf{b}-(1-\sigma) \mathbf{A}_{e}^{+} \mathbf{b}_{e}
\end{aligned}
$$

with $\sigma$ a continuous transition function equal to 0 in the neighborhood of a singularity and to 1 away from it.

$$
\sigma=\left\{\begin{aligned}
1 & \text { if } \frac{1}{\kappa(\mathbf{A})}>2 \varepsilon \\
\frac{1-\kappa(\mathbf{A}) \varepsilon}{\kappa(\mathbf{A}) \varepsilon} & \text { if } \varepsilon<\frac{1}{\kappa(\mathbf{A})}<2 \varepsilon \\
0 & \text { if } \frac{1}{\kappa(\mathbf{A})}<\varepsilon
\end{aligned}\right.
$$

with $\varepsilon$ a threshold characterizing the proximity of the singularity determined experimentally.

\subsubsection{LPJTS singularity}

A similar result can be obtained for a LPJTS singularity. The equation (27) cannot be solved at singularity locus. In this case, the extended second-order loop-closure equation will take the form

$$
\mathbf{J}_{d, e} \ddot{\mathbf{q}}_{d}=-\mathbf{J}_{d a, e} \ddot{\mathbf{q}}_{a}-\mathbf{n}_{e}
$$

with

$$
\begin{gathered}
\mathbf{J}_{d, e}=\left[\begin{array}{c}
\mathbf{J}_{d} \\
\dot{\mathbf{q}}_{d s}^{T} \mathbf{M}_{d e}
\end{array}\right] \quad \mathbf{J}_{d a, e}=\left[\begin{array}{c}
\mathbf{J}_{d a} \\
\mathbf{0}_{1 \times n_{d o f}}
\end{array}\right] \quad \mathbf{n}_{e}=\left[\begin{array}{c}
\mathbf{n} \\
\dot{\mathbf{q}}_{d s}^{T} \mathbf{c}_{d e}
\end{array}\right] \\
\mathbf{M}_{d e}=\mathbf{M}_{d}\left[\begin{array}{c}
-\mathbf{J}_{d a}^{-1} \mathbf{J}_{d} \\
\mathbf{1}_{n_{p} \times 1}
\end{array}\right] \quad \mathbf{c}_{d e}=\mathbf{c}_{d}+\mathbf{M}_{d}\left[\begin{array}{c}
-\mathbf{J}_{d a}^{-1} \mathbf{n} \\
\mathbf{0}_{n_{p} \times 1}
\end{array}\right]
\end{gathered}
$$

With a reasoning similar to the one applied for Type 2 singularities (section (3.1.1)), it can be proved that the system (40) will give a unique solution in LPJTS singularity. The computation of the passive joint acceleration is then a combination of the classical loop-closure equations (30) and the extended loop-closure equation (40),

$$
\begin{aligned}
\ddot{\mathbf{q}}_{d}= & -\left(\sigma_{p} \mathbf{J}_{d}^{-1} \mathbf{J}_{d a}-\left(1-\sigma_{p}\right) \mathbf{J}_{d, e}^{+} \mathbf{J}_{d a, e}\right) \ddot{\mathbf{q}}_{a} \\
& -\sigma_{p} \mathbf{J}_{d}^{-1} \mathbf{n}-\left(1-\sigma_{p}\right) \mathbf{J}_{t d, e}^{+}, \mathbf{n}_{e}
\end{aligned}
$$

where

$$
\sigma_{p}=\left\{\begin{array}{c}
1 \text { if } \frac{1}{\kappa\left(\mathbf{J}_{d}\right)}>2 \varepsilon_{p}, \\
\frac{1-\kappa\left(\mathbf{J}_{d}\right) \varepsilon_{p}}{\kappa\left(\mathbf{J}_{d}\right) \varepsilon_{p}} \text { if } \varepsilon<\frac{1}{\kappa\left(\mathbf{J}_{d}\right)}<2 \varepsilon_{p} \\
0 \text { if } \frac{1}{\kappa\left(\mathbf{J}_{d}\right)}<\varepsilon_{p}
\end{array}\right.
$$

with $\varepsilon_{p}$ a threshold characterizing the proximity of the singularity.

\subsection{Expression of the inverse dynamic model for a con- trol in joint space}

In section 2.3.1, general solutions to the inverse dynamic model that are valid in and out of a singularity are given by equation (18) for Type 2 singularities and (24) for LPJTS singularities. Those models will be used for the design of a the computed torque control law to cross the singularity. The inverse dynamic model is expressed as function of the vectors $\tau_{a}, \tau_{d}$ and $\mathbf{w}_{x}$ issued from the Lagrangian of the system by equations (3)-(5). For encoder-based computed torque control purpose, this inverse dynamic model must be expressed as a function of the acceleration of the measured coordinates $\ddot{\mathbf{q}}_{a}$. This expression will be computed using equations (9)(11) and the extended second-order loop-closure equations expressed in section 3.1.

\subsubsection{Type 2 singularity}

Combining the second order kinematic equations (27) and (38) with the equations issued from the Lagrangian (11)(10) and the inverse dynamic model (18) leads to the expression of the model as function of the joint acceleration $\ddot{\mathbf{q}}_{a}$. The model can be expressed under the form

$$
\tau=\mathbf{M}_{T 2} \ddot{\mathbf{q}}_{a}+\mathbf{h}_{T 2}
$$

where

- $\mathbf{M}_{T 2}$ is the $\left(n_{d o f} \times n_{d o f}\right)$ inertia matrix of the system. $\mathbf{M}_{T 2}$ depends on the joints coordinates $\mathbf{q}$ and platform coordinates $\mathbf{x}$. Skipping all mathematical derivations, it comes

$$
\begin{aligned}
\mathbf{M}_{T 2} & =\mathbf{J}_{T 2}^{T}{ }^{+}\left(\mathbf{J}_{T 2}^{T} \mathbf{M}_{a} \mathbf{J}_{\sigma}\right. \\
& +\mathbf{M}_{x}\left(\sigma \mathbf{A}^{-1} \mathbf{B}+(1-\sigma) \mathbf{A}_{e}^{+} \mathbf{B}_{e}\right) \\
& \left.-\left(\mathbf{J}_{x}^{T}+\mathbf{J}_{T 2}^{T} \mathbf{J}_{a}^{T}\right) \mathbf{J}_{d}^{-T} \mathbf{J}_{\sigma}\right)
\end{aligned}
$$

$$
\text { with } \mathbf{J}_{\sigma}=\left[\begin{array}{c}
\mathbf{I}_{n_{d o f}} \\
-\mathbf{J}_{d}^{-1}\left(\mathbf{J}_{x}\left(\sigma \mathbf{A}^{-1} \mathbf{B}+(1-\sigma) \mathbf{A}_{e}^{+} \mathbf{B}_{\mathbf{e}}\right)+\mathbf{J}_{a}\right)
\end{array}\right] .
$$

- $\mathbf{h}_{T 2}$ is a $n_{d o f}$-dimensional vector regrouping the gravitational, centrifugal and Coriolis terms. $\mathbf{h}_{T 2}$ depends on the joints coordinates $\mathbf{q}$ and velocities $\dot{\mathbf{q}}$ and platform 
coordinates $\mathbf{x}$ and their time derivative $\dot{\mathbf{x}}$. Skipping all mathematical derivations, one can obtain

$$
\begin{aligned}
\mathbf{h}_{T 2} & =\mathbf{J}_{T 2}^{T}+\left(\mathbf{J}_{T 2}^{T}\left(\mathbf{M}_{a} \mathbf{n}_{\sigma}+\mathbf{c}_{a}\right)\right. \\
& +\mathbf{M}_{x}\left(\sigma \mathbf{A}^{-1} \mathbf{b}+(1-\sigma) \mathbf{A}_{e}^{+} \mathbf{b}_{e}\right)-\mathbf{c}_{x} \\
& \left.-\left(\mathbf{J}_{x}^{T}+\mathbf{J}_{T 2}^{T} \mathbf{J}_{a}^{T}\right) \mathbf{J}_{d}^{-T}\left(\mathbf{M}_{d} \mathbf{n}_{\sigma}-\mathbf{c}_{d}\right)\right)
\end{aligned}
$$

$$
\text { with } \mathbf{n}_{\sigma}=\left[\begin{array}{c}
\mathbf{0}_{n_{d o f} \times 1} \\
-\mathbf{J}_{d}^{-1} \mathbf{J}_{x}\left(\sigma \mathbf{A}^{-1} \mathbf{b}+(1-\sigma) \mathbf{A}_{e}^{+} \mathbf{b}_{e}\right)+\mathbf{d}
\end{array}\right] .
$$

\subsubsection{LPJTS singularity}

An expression similar to equation (43) can be derived from the inverse dynamic model obtained for a LPJTS singularity. Combining the second order kinematic equations (29) and (41) with the equations issued from the Lagrangian (11)-(10) and the inverse dynamic model equation (24) leads to the expression of the model as function of the joint acceleration $\ddot{\mathbf{q}}_{a}$.

$$
\tau=\mathbf{M}_{p} \ddot{\mathbf{q}}_{a}+\mathbf{h}_{p}
$$

Skipping the mathematical derivations, $\mathbf{M}_{p}$ is given by

$$
\begin{aligned}
\mathbf{M}_{p} & =\mathbf{J}_{p}^{T+}\left(-\mathbf{M}_{d} \mathbf{J}_{\sigma_{p}}\right. \\
& \left.+\mathbf{J}_{p}^{T}\left(\mathbf{M}_{a} \mathbf{J}_{\sigma_{p}}+\mathbf{B}^{T} \mathbf{A}^{-T} \mathbf{M}_{x} \mathbf{A}^{-1} \mathbf{B}\right)\right)
\end{aligned}
$$

with $\mathbf{J}_{\sigma p}=\left[\begin{array}{c}\mathbf{I}_{n_{d o f}} \\ -\sigma_{p} \mathbf{J}_{d}^{-1} \mathbf{J}_{d a}-\left(1-\sigma_{p}\right) \mathbf{J}_{t d, e}^{+} \mathbf{J}_{e}\end{array}\right]$ and $\mathbf{h}_{p}$ is given by

$$
\begin{aligned}
\mathbf{h}_{p} & =\mathbf{J}_{p}^{T+}\left(-\left(\mathbf{M}_{d} \mathbf{n}_{\sigma_{p}}+\mathbf{c}_{d}\right)+\mathbf{J}_{p}^{T}\left(\mathbf{M}_{a} \mathbf{n}_{\sigma_{p}}+\mathbf{c}_{a}\right.\right. \\
& \left.\left.+\mathbf{B}^{T} \mathbf{A}^{-T}\left(\mathbf{M}_{x} \mathbf{A}^{-1} \mathbf{b}-\mathbf{c}_{r}\right)\right)\right)
\end{aligned}
$$

with $\mathbf{n}_{\sigma p}=\left[\begin{array}{c}\mathbf{0}_{n_{d o f} \times 1} \\ -\sigma_{p} \mathbf{J}_{d}^{-1} \mathbf{n}-\left(1-\sigma_{p}\right) \mathbf{J}_{t d, e}^{+} \mathbf{n}_{e}\end{array}\right]$

\subsection{Wrench projection in the neighborhood of a singu- larity}

Around the singularity locus, the dynamic criterion established (Table 1) will not be exactly respected by a controller in real conditions. Unfortunately, only the strict respect of this criterion guarantee an exact solution to the inverse dynamic model. The existence of numerical and tracking errors may lead to the computation of huge torques in the neighborhood of a singularity. Error amplification around the singularity is due to the presence of a singular value near zero in the inverted matrix. Based on the assumption that the criterion is respected in the neighborhood of the singularity, a projection of the desired wrench in the dynamic model is performed. The design of the error inhibitor is given in this section for a Type 2 singularity from equation (18). The design of a similar inhibitor for a LPJTS singularity can be obtained from equation (24).

Let us consider the inverse dynamic model equation to solve under the form (18)

$$
\mathbf{A}^{T} \mathbf{B}^{-T} \tau=\mathbf{w}_{T 2}
$$

The matrix A degenerates in a Type 2 singularity. Let consider its singular value decomposition

$$
\mathbf{A}=\mathbf{U} \Sigma \mathbf{V}^{T}
$$

where $\Sigma$ is the diagonal matrix of ordered positive singular values $\left(e_{1}, \ldots, e_{n}\right)$ and $\mathbf{U}, \mathbf{V}$ two unitary matrices. At singularity locus, the last singular value is null $e_{n}=0$. Multiplying equation (49) by the matrix $\mathbf{V}^{T}$ gives

$$
\Sigma \mathbf{U}^{T} \mathbf{B}^{-T} \tau=\mathbf{V}^{T} \mathbf{w}_{T 2}
$$

In order for the dynamic model to give an exact solution, the last component of the vector $\mathbf{w}_{S}=\mathbf{V}^{T} \mathbf{w}_{T 2}$ must be equal to zero. This is an other formulation of the dynamic criterion necessary to cross the singularity (note that the last line of the matrix $\mathbf{V}^{T}$ is included in $\operatorname{ker} \mathbf{A}$ ). As the dynamic criterion is not exactly respected, the last component of the vector $\mathbf{w}_{S}$ has to be driven toward zero in the neighborhood of the singularity to avoid the numerical degeneracy of the inverse dynamic model. As in section 3.1.1, the proximity to the singularity is characterized by the condition number using the norm-2 of the matrix A. A function $\sigma_{s}$ is defined by

$$
\sigma_{s}=\left\{\begin{array}{c}
1 \text { if } \frac{1}{\kappa(\mathbf{A})}>2 \varepsilon_{s}, \\
\frac{1-\kappa(\mathbf{A}) \varepsilon_{s}}{\kappa(\mathbf{A}) \varepsilon_{s}} \text { if } \varepsilon_{s}<\frac{1}{\kappa(\mathbf{A})}<2 \varepsilon_{s} \\
0 \text { if } \frac{1}{\kappa(\mathbf{A})}<\varepsilon_{s} .
\end{array}\right.
$$

with $\varepsilon_{s}$ threshold determined experimentally. $\sigma_{s}$ is a function equals to 1 and continuously driven towards 0 as the system get closer to the singularity. Multiplying the last component of the vector $\mathbf{w}_{s}$ by $\sigma_{s}$ ensures that the desired wrench in equation (49) respects the dynamic criterion. This will avoid exponential increase of the torques consequent to minimal errors on the trajectory tracking.

\subsection{Computed torque control law}

The computed torque control (CTC) is an advanced control law for non-linear systems where the inverse dynamic model is used to realize an exact linearization of the system. Computed torque control allows the design of considerably more precise, energy-efficient and compliant controls for robots. The inclusion of the mechanism dynamic model 


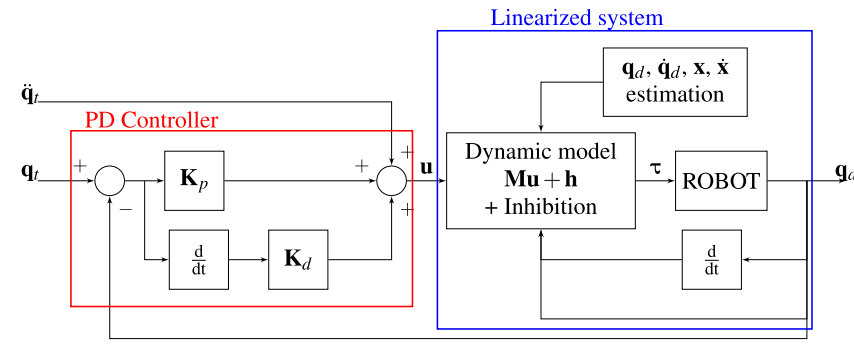

Figure 2: Computed Torque controller in joint space

into the controller is desirable to enforce a dynamical criterion such as the criterion required for singularity crossing (Table 1). The convergence and the stability of such controllers is well known [22]. For all aforementioned reasons, this control law is an excellent candidate to track trajectories crossing the singularity. The following developments are suited both for Type 2 and LPJTS singularities.

From the previous section results, the inverse dynamic model of the robot can be expressed as a function of the active joint accelerations even at singular configuration $\ddot{\mathbf{q}}_{a}$ :

$$
\tau=\mathbf{M} \ddot{\mathbf{q}}_{a}+\mathbf{h}
$$

The computed torque control law is obtained with the design of an auxiliary control signal $\mathbf{u}$ corresponding to the joint acceleration $\ddot{\mathbf{q}}_{a}$ from which the input torques are computed

$$
\tau=\mathbf{M u}+\mathbf{h}
$$

The following PD control law is applied

$$
\mathbf{u}=\ddot{\mathbf{q}}_{t}+\mathbf{K}_{d}\left(\dot{\mathbf{q}}_{t}-\dot{\mathbf{q}}_{a}\right)+\mathbf{K}_{p}\left(\mathbf{q}_{t}-\mathbf{q}_{a}\right)
$$

where

- $\mathbf{q}_{t}, \dot{\mathbf{q}}_{t}$ and $\ddot{\mathbf{q}}_{t}$ are respectively the desired active joints position, velocity and acceleration along the tracked trajectory,

- $\mathbf{K}_{d}$ and $\mathbf{K}_{p}$ are positive definite gain matrices.

This control law ensures a second-order convergence of the joint position error $\mathbf{e}=\mathbf{q}_{t}-\mathbf{q}_{a}$. Fig. 2 represents the classical CTC controller that can be applied to a parallel mechanism in order to track a trajectory. The computation of the matrices $\mathbf{M}$ and $\mathbf{h}$ requires an estimation of the platform and passive joints position and velocity. Several techniques may be used to get this estimation such as a computation through the geometric model, interval analysis method [23] or exteroceptive measures [24]. The estimation may also be computed from the desired trajectory, as long as the tracking is reliable [19]. A computed torque control in Cartesian coordinates may also be designed to limit the issues with solutions to the second-order kinematic equations for Type 2 singularities (see section 3.1.1). However, to be efficient, such controller requires a precise external measure of the end-effector

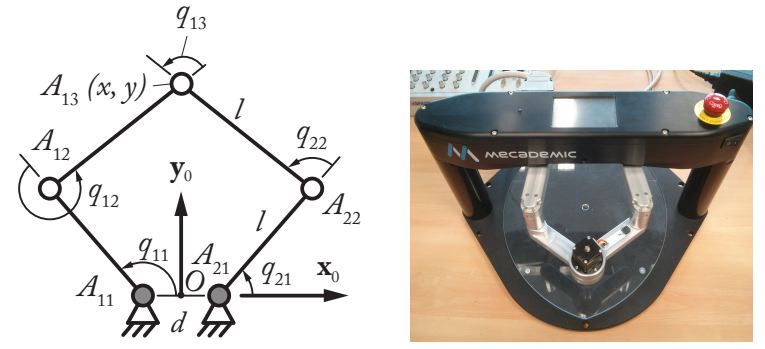

Figure 3: Five-bar mechanism and parametrization scheme

position, which is currently rarely available on robots. For a review of dynamic control of parallel robots, the reader is referred to [25].

\section{Case study}

The proposed approach was validated on an experimental platform. As the models and controllers are similar for Type 2 and LPJTS singularities, the experimental validation is only presented for the crossing of Type 2 singularities with a planar five-bar mechanism.

A five-bar mechanism is a planar parallel mechanism composed of two actuated joints located points $A_{11}$ and $A_{21}$ and three passive joints located at points $A_{12}, A_{22}$ and $A_{13}$ (see Fig. 3). This mechanism is able to generate a motion of the end-effector situated in $A_{13}\left(\mathbf{x}=(x, y)^{T}\right)$ through the actuation of the joints $q_{11}$ and $q_{21}$. The passive joints coordinates are respectively noted $q_{12}$ on the first arm, $q_{22}$ on the second arm and $q_{13}$ for the joint linking those two arms.

\subsection{Identified dynamic model}

A full dynamic model identification was computed using a weighted least square method applied on exciting trajectories described in $[26,27]$. The base parameters identified for the five-bar mechanism were

- grouped inertia for the two proximal links $z z_{11 R}=0.0133 \mathrm{~kg} / \mathrm{m}^{2}$ and $z z_{21 R}=0.0142 \mathrm{~kg} / \mathrm{m}^{2}$,

- grouped point mass at the end-effector $m_{3 R}=0.537 \mathrm{~kg}$,

- frictions (coulomb model) static $f_{o f f 11}=0.0855 \mathrm{Nm}$, $f_{\text {off } 21}=0 \mathrm{Nm}$ and kinetic $f_{s 11}=0.345 \mathrm{Nm} / \mathrm{s}, f_{s 21}=$ $0.430 \mathrm{Nm} / \mathrm{s}$ parameters for the active joints.

The base parameters lead to the computation of the dynamic model (6)-(8) under the form

$$
\left\{\begin{array}{l}
\tau=\mathbf{Z} \ddot{\mathbf{q}}_{a}+\mathbf{f}-\mathbf{B}^{T} \boldsymbol{\lambda}_{1} \\
\mathbf{A}^{T} \lambda_{1}=m_{3 R} \ddot{\mathbf{x}}
\end{array}\right.
$$

with

- $\ddot{\mathbf{q}}_{a}=\left(\ddot{q}_{11}, \ddot{q}_{21}\right)^{T}$ the active joint accelerations vector,

- $\mathbf{A}$ and $\mathbf{B}$ issued from the loop-closure equations (1) and (2), 


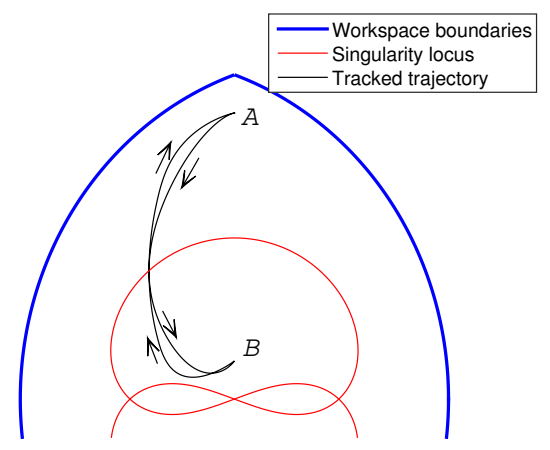

Figure 4: Tracked trajectory in Cartesian space (to scale)

- $\mathbf{Z}$ a positive diagonal matrix

$$
\mathbf{Z}=\left[\begin{array}{cc}
z z_{11 R} & 0 \\
0 & z z_{21 R}
\end{array}\right]
$$

- $\mathbf{f}$ the active joint friction terms

$$
\left[\begin{array}{l}
f_{s 11} \operatorname{sign}\left(\dot{q}_{11}\right) \\
f_{s 21} \operatorname{sign}\left(\dot{q}_{21}\right)
\end{array}\right]+\left[\begin{array}{l}
f_{o f f 11} \\
f_{o f f 21}
\end{array}\right]
$$

with sign a mathematical function extracting the sign of a real number.

The five-bar mechanism presents Type 2 singularities corresponding to the degeneracy of the matrix A. Type 2 singularity configurations happen when the two distal links of the five-bar mechanism are aligned. In this case, the uncontrolled motion is orthogonal to this alignment (see Fig. 1). The dynamic criterion to be applied when crossing a Type 2 singularity (Table 1), related to the dynamic model (56), is then given by the equation

$$
\ddot{y}=\ddot{x} \tan \left(q_{i 1, s}+q_{i 2, s}\right)
$$

with $q_{i 1, s}$ and $q_{i 2, s}$ joint $i 1$ and $i 2$ positions of arm $i$ in singular configuration. The expression of the inverse dynamic model that does not degenerate at singularity locus (18), as long as the criterion (59) is respected, is given by

$$
\tau=\left(\mathbf{J}_{T 2}^{T}\right)^{+}\left(\mathbf{J}_{T 2}^{T} \mathbf{Z} \ddot{\mathbf{q}}_{a}-m_{3 R} \ddot{\mathbf{x}}\right)
$$

\subsection{Trajectory generation}

To test the controller a trajectory crossing twice a Type 2 singularity has been generated with the five-bar mechanism. This trajectory (shown in Fig. 4) is a return trip from a point $A=[0 ; 0.15] \mathrm{m}$ to a point $B=[0 ; 0.02] \mathrm{m}$. The trajectory crosses twice the singularity point $S=[-0.048 ; 0.067] \mathrm{m}$. A point to point trajectory is computed in Cartesian coordinates and composed of
- A trajectory during a time interval $\left[t_{s i} ; t_{s o}\right]$ around the singularity with constant acceleration $\left(\ddot{x}_{s}, \ddot{y}_{s}\right)$ that respects the criterion (59), given by (with $t$ the time variable)

$$
\begin{aligned}
& x=\frac{\ddot{x}_{s}}{2} t^{2}+\alpha_{x} t+\beta_{x} \\
& y=\frac{\ddot{y}_{s}}{2} t^{2}+\alpha_{y} t+\beta_{y}
\end{aligned}
$$

$\alpha_{x}, \alpha_{y}, \beta_{x}$ and $\beta_{y}$ are coefficients computed such that the trajectory respect a desired position and velocity at singularity point.

- Two five degree polynomials ensuring a continuous transition form the starting point to the singularity trajectory and from the singularity trajectory to the end point. Those polynomials are defined by initial and final position, a null velocity and null acceleration of the endeffector at those points and by the the trajectory duration $t_{f}$.

Table 2 gives the parameters used to define the trajectory from point $A$ to point $B$ and from point $B$ to point $A$. The trajectory in joint space is computed through the inverse geometric model of the robot.

Table 2: Trajectory parameters

\begin{tabular}{c|c|c|c|c|c|c|c|c} 
& $\begin{array}{c}t_{f} \\
{[\mathrm{sec}]}\end{array}$ & $\begin{array}{c}t_{s} \\
{[\mathrm{sec}]}\end{array}$ & $\begin{array}{c}t_{s i} \\
{[\mathrm{sec}]}\end{array}$ & $\begin{array}{c}t_{s o} \\
{[\mathrm{sec}]}\end{array}$ & $\begin{array}{c}\dot{x}_{s} \\
{[\mathrm{~m} / \mathrm{s}]}\end{array}$ & $\begin{array}{c}\dot{y}_{s} \\
{[\mathrm{~m} / \mathrm{s}]}\end{array}$ & $\begin{array}{c}\ddot{x}_{s} \\
{\left[\mathrm{~m} / \mathrm{s}^{2}\right]}\end{array}$ & $\begin{array}{c}\ddot{y}_{s} \\
{\left[\mathrm{~m} / \mathrm{s}^{2}\right]}\end{array}$ \\
\hline$A \rightarrow B$ & 0.7 & 0.35 & 0.25 & 0.45 & 0 & -0.374 & 2 & 0.469 \\
$B \rightarrow A$ & 1.4 & 1.15 & 1.05 & 1.25 & 0 & -0.374 & 1 & 0.234
\end{tabular}

\subsection{Experimental results}

This section presents the experimental results of the trajectory defined in section 4.2. Figure 5 shows the torque computed by the controller and the joint tracking error during the trajectory. The thresholds $\varepsilon$ and $\varepsilon_{s}$ are both set to 0.1 for this experiment. With the dynamic model computed, there is no discontinuity in torque computation when crossing the singularity. The tracking error may locally increase just after the singularity crossing. Several factors generate this effect. First, the dynamic model around the singularity is an approximation which considers that the criterion established (59) is strictly respected. In real conditions, a strict respect of the criterion cannot be ensured, leading to a less efficient estimation of the dynamic model around the singularity and a deterioration of the tracking quality. It must also be considered that locally, a Type 2 singularity constraints the gap between the two proximal legs of the system, if the calibration of the proximal joint positions is not of perfect accuracy, an error will be generated by this constraint when crossing. And finally, it must be considered that in the neighborhood of a singularity, the system is locally underactuated. 

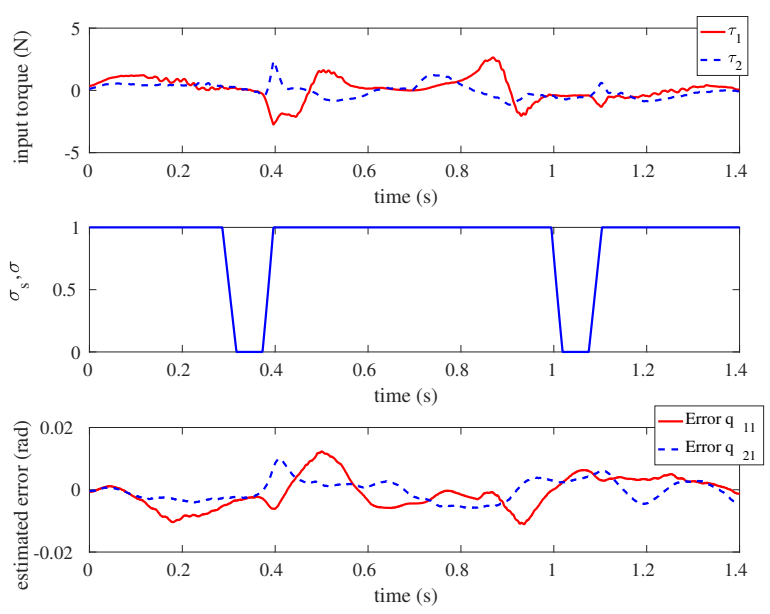

Figure 5: Input torque applied, $\sigma$ and $\sigma_{s}$ values and tracking error on a singularity crossing trajectory. $\sigma=\sigma_{s}=0$ means that the robot is close to a singularity and the wrench projection in the controller is active.

This aspect makes a part of the tracking errors locally impossible to be corrected by the controller. The appropriate precautions taken make that the torque input remain bounded at singularity, at the cost of a local angular tracking error.

Nevertheless, a trajectory have been generated with several singularity points and accelerations. The trajectory have been tested at least 10 times with success in crossing the singularities each time. Experiment videos are available at https://www.youtube.com/watch?v=xVAaF4ycfho

\section{Conclusion}

The presence of singularities in the workspace of parallel robot reduces the effective workspace. To increase this workspace, a promising solution that consists in designing trajectories able to cross singularities has been developed in a previous study. However, the controllers developed to track those trajectories were limited to restrictive cases of Type 2 singularities crossing. This paper proposes new expressions of the inverse dynamic model, adapted for computation even in the singularity. The inverse dynamic model developed also handle the local overconstraint that appears in the singularity. Combined with the extension of the second order loopclosure equations in the singularity, it allows the design of a computed torque control able to track the desired trajectories. The theoretical contributions of this paper also extended the results to the crossing of LPJTS singularities. The controller was validated experimentally on a five-bar mechanism.

Future work on this subject includes the development of techniques to validate the operating mode change when crossing a singularity without the use of exteroceptive sensors. Also, one limitation of the controller is its dependency to a threshold determined experimentally. An improvement of the approach would be to develop a formal method to compute an appropriate value. Work related to a better consider- ation of the underactuation into the controller when crossing the singularity is also in progress.

\section{References}

[1] Merlet, J., 2006. Parallel robots. Springer- ISBN: 9781-4020-4133-4.

[2] Zlatanov, D., Fenton, R., and Benhabib, B., 1994. "Singularity analysis of mechanisms and robots via a velocity-equation model of the instantaneous kinematics". In Proceedings of IEEE International Conference on Robotics and Automation, pp. 986-991.

[3] Zlatanov, D., Bonev, I. A., and Gosselin, C. M., 2002. "Constraint singularities of parallel mechanisms". In Proceedings of IEEE International Conference on Robotics and Automation, pp. 496-502.

[4] Conconi, M., and Carricato, M., 2009. "A New Assessment of Singularities of Parallel Kinematic Chains". IEEE Transactions on Robotics, 25(4), aug, pp. 757770.

[5] Briot, S., Pagis, G., Bouton, N., and Martinet, P., 2015. "Degeneracy conditions of the dynamic model of parallel robots". Multibody System Dynamics, oct, pp. 1-42.

[6] Gosselin, C., and Angeles, J., 1990. "Singularity analysis of closed-loop kinematic chains". IEEE Transactions on Robotics and Automation, 6(3), jun, pp. 281290.

[7] Liu, X.-J., Wang, J., and Pritschow, G., 2006. "Kinematics, singularity and workspace of planar 5R symmetrical parallel mechanisms". Mechanism and Machine Theory, 41(2), feb, pp. 145-169.

[8] Gogu, G., 2004. "Structural synthesis of fully-isotropic parallel robots via theory of linear transformations and evolutionary morphology". European Journal of Mechanics, A/Solids, 23, pp. 1021-1039.

[9] Briot, S., and Arakelian, V., 2010. "On the Dynamic Properties of Rigid-Link Flexible-Joint Parallel Manipulators in the Presence of Type 2 Singularities". ASME. J. Mechanisms Robotics., 2(2), pp. 021004, 6 pages.

[10] Nahon, M. A., and Angeles, J., 1989. "Force optimization in redundantly-actuated closed kinematic chains". In Proceedings of IEEE International Conference on Robotics and Automation, pp. 951-956.

[11] Kurtz, R., and Hayward, V., 1992. "Multiple-goal kinematic optimization of a parallel spherical mechanism with actuator redundancy". IEEE Transactions on Robotics and Automation, 8(5), pp. 644-651.

[12] Rakotomanga, N., Chablat, D., and Caro, S., 2008. Kinetostatic Performance of a Planar Parallel Mechanism with Variable Actuation. Springer - ISBN: 9781-402-04940-8.

[13] Arakelian, V., Briot, S., and Glazunov, V., 2008. "Increase of singularity-free zones in the workspace of parallel manipulators using mechanisms of variable structure". Mechanism and Machine Theory, 43(9), sep, pp. 1129-1140.

[14] Campos, L., Bourbonnais, F., Bonev, I. A., and Bigras, P., 2010. "Development of a Five-Bar Parallel Robot 
With Large Workspace”. In Proceedings of Annual Mechanisms and Robotics Conference, pp. 917-922.

[15] Zein, M., Wenger, P., and Chablat, D., 2008. "NonSingular Assembly-mode Changing Motions for 3RPR Parallel Manipulators". Mechanism and Machine Theory, 43(4), mar, pp. 480-490.

[16] Ider, S. K., 2005. "Inverse dynamics of parallel manipulators in the presence of drive singularities". Mechanism and Machine Theory, 40(1), jan, pp. 33-44.

[17] Pagis, G., Bouton, N., Briot, S., and Martinet, P., 2015. "Enlarging parallel robot workspace through Type-2 singularity crossing". Control Engineering Practice, 39, jun, pp. 1-11.

[18] Six, D., Briot, S., Chriette, A., and Martinet, P., 2016. "A Controller for Avoiding Dynamic Model Degeneracy of Parallel Robots During Type 2 Singularity Crossing”. In New Trends in Mechanism and Machine Science (EUCOMES proc.), pp. 589-597.

[19] Khalil, W., and Dombre, E., 2002. Modeling, Identification and Control of Robots. Kogan Page Science.

[20] Briot, S., and Khalil, W., 2015. Dynamics of Parallel Robots: From Rigid Bodies to Flexible Elements. Springer - ISBN: 978-3-319-19787-6.

[21] Briot, S., and Arakelian, V., 2008. “Optimal Force Generation in Parallel Manipulators for Passing through the Singular Positions". The International Journal of Robotics Research, 27(8), pp. 967-983.

[22] Samson, C., 1987. "Robust control of a class of nonlinear systems and applications to robotics". International Journal of Adaptive Control and Signal Processing, 1, pp. 49-68.

[23] Merlet, J. P., 2004. "Solving the Forward Kinematics of a Gough-Type Parallel Manipulator with Interval Analysis". The International Journal of Robotics Research, 23(3), mar, pp. 221-235.

[24] Özgür, E., Dahmouche, R., Andreff, N., and Martinet, P., 2014. "A vision-based generic dynamic model of PKMs and its experimental validation on the Quattro parallel robot". In Proceedings of IEEE/ASME International Conference on Advanced Intelligent Mechatronics, pp. 937-942.

[25] Paccot, F., Andreff, N., and Martinet, P., 2009. "A Review on the Dynamic Control of Parallel Kinematic Machines: Theory and Experiments". The International Journal of Robotics Research, 28(3), pp. 395416.

[26] Gautier, M., Khalil, W., and Restrepo, P. P., 1995. "Identification of the dynamic parameters of a closed loop robot". In Proceedings of IEEE International Conference on Robotics and Automation, pp. 3045-3050.

[27] Renaud, P., Vivas, A., Andreff, N., Poignet, P., Martinet, P., Pierrot, F., and Company, O., 2006. "Kinematic and dynamic identification of parallel mechanisms". Control Engineering Practice, 14(9), sep, pp. 1099-1109.

\section{List of Figures}

1 Example of a five-bar mechanism in a Type 2 singularity configuration ....... . 3

2 Computed Torque controller in joint space . . 8

3 Five-bar mechanism and parametrization scheme .............. . . 8

4 Tracked trajectory in Cartesian space (to scale) 9

5 Input torque applied, $\sigma$ and $\sigma_{s}$ values and tracking error on a singularity crossing trajectory .............. 10

\section{List of Tables}

1 Dynamic criterion to avoid degeneracy of the inverse dynamic model in a singularity . . . . 3

2 Trajectory parameters ......... 9 